\title{
Electron Beam-Induced Object Excitations at Atomic Resolution - Minimization and Exploitation
}

\author{
Christian Kisielowski ${ }^{1}$, H.A. Calderon ${ }^{2}$, Stig Helveg ${ }^{3}$, Petra Specht ${ }^{4}$ \\ 1. LBNL, The Molecular Foundry, One Cyclotron Road, Berkeley California, USA. \\ 2. Departamento de Física, ESFM-IPN, Ed. 9, UPALM-Zacatenco, México, D.F., Mexico \\ 3. Haldor Topsoe A/S, Haldor Topsøes Allé 1, DK-2800, Kgs. Lyngby, Denmark \\ 4. Department of Materials Science \& Engineering, UC Berkeley, Berkeley, CA, USA
}

Over the last decade it became evident that atomic resolution electron microscopy allows reliably analyzing matter down to single atoms as long as electron beam-induced object alterations remain negligible. In general, however, nano-objects are unavoidably altered by the intense electron irradiation while acquiring atomic resolution images with single atom sensitivity. With efforts rapidly growing to enable atomic resolution imaging of hybrid (soft and hard) matter and even biological objects the control of electron dose and dose rates as well as acceleration voltages are no longer a commodity but have become a necessity.

We have addressed the existing bottlenecks [1] by developing low dose-rate in-line holography, which efficiently captures all details of the elastic scattering process by recovering electron exit wave functions in a fully quantitative manner [2]. Research directions that utilize the tool cut two ways: On one hand it has become possible to better maintain the atomic structure of radiation sensitive matter by integrating dose rates as low as $1-100 \mathrm{e} / \AA^{2} \mathrm{~s}$ into the image acquisition process that are known to even maintain structural integrity of biological objects. In such conditions, single atomic resolution images are necessarily noise dominated (Figure 1a) but the acquisition and analysis of image series allows for a faithful restoration of the atomic structure (Figure 1b, c, e). The outstanding contrast in such images can quantitatively be matched with element specificity (Figure 1d) including 3D [3]. On the other hand a systematic increase of dose rates and total doses uncovers the onset of dynamic responses of the system. For example, collective atom displacements in graphene were predicted by molecular dynamics simulations at $\mathrm{Tm} / 4, \mathrm{Tm}=$ melting point temperature [4] that can be described by a cumulated Rayleigh distribution function (Figure 2a). Deviations from the distribution occur at higher temperatures (Figure $2 b)$. They are caused by reversible, collective atom excitations that can be observed in experimental images as large atom displacements (Figure 2c) that overwrite predictions made by Debye-Waller factors. The effect usually causes a significant contrast broadening in $2 \mathrm{D}$ materials (Figure $2 \mathrm{~d}$ ). Recent, successful applications of the tool include the discovery of multifunctional biphasic water splitting catalyst that is tailored for integration with high-performance semiconductor photoanodes [5] and the atomic resolution imaging of radiation sensitive halide perovskites [6], which can be characterized atom-by-atom in terms of chemical composition and local structure. [7]

\section{References:}

[1] G.A. Botton, H.A. Calderon and C. Kisielowski, Micron 68 (2015), p. 140.

[2] B. Barton et al, Microscopy and Microanalysis 18 (2012), p. 982.

[3] F-R. Chen, D. Van Dyck, C. Kisielowski, Nature Com. 7, (2016), p.10603.

[4] C. Kisielowski et al, Phys Rev. B 88 (2013), p. 024305.

[5] J. Yang et al, Nature Materials (2016) DOI: 10.1038/NMAT4794 
[6] Y. Yu et al, Nano Letters (2016) DOI: 10.1021/acs.nanolett.6b03331

[7] The Molecular Foundry is supported by the Office of Science, the Office of Basic Energy Sciences, the U.S. Department of Energy under Contract No. DE-AC02-05CH11231.
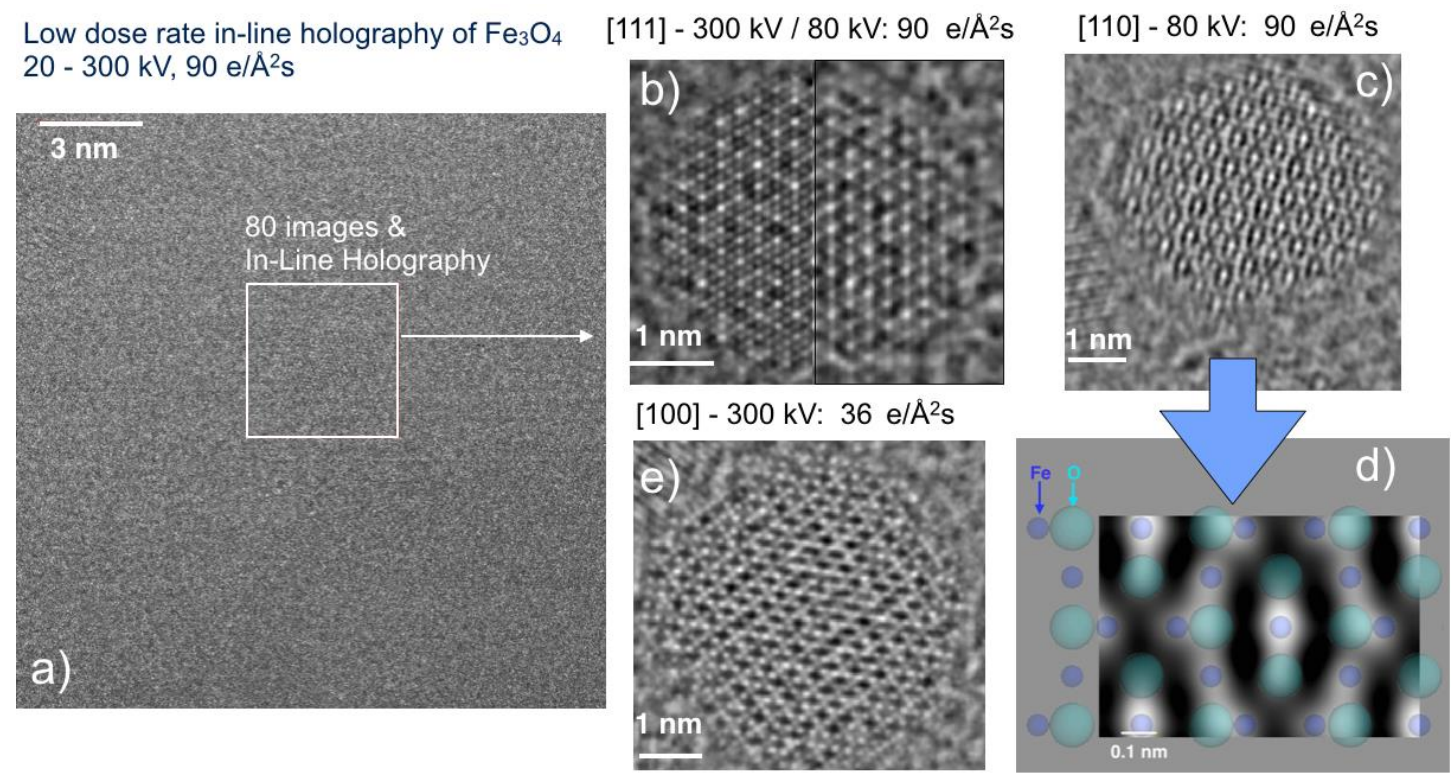

Figure 1. Maintaining structural integrity by low dose-rate in-line holography [2]: a) single image; b, c, e) reconstructed phase images of $\mathrm{Fe}_{3} \mathrm{O}_{4}$ catalysts in 3 zone axes orientations. c) chemical composition.
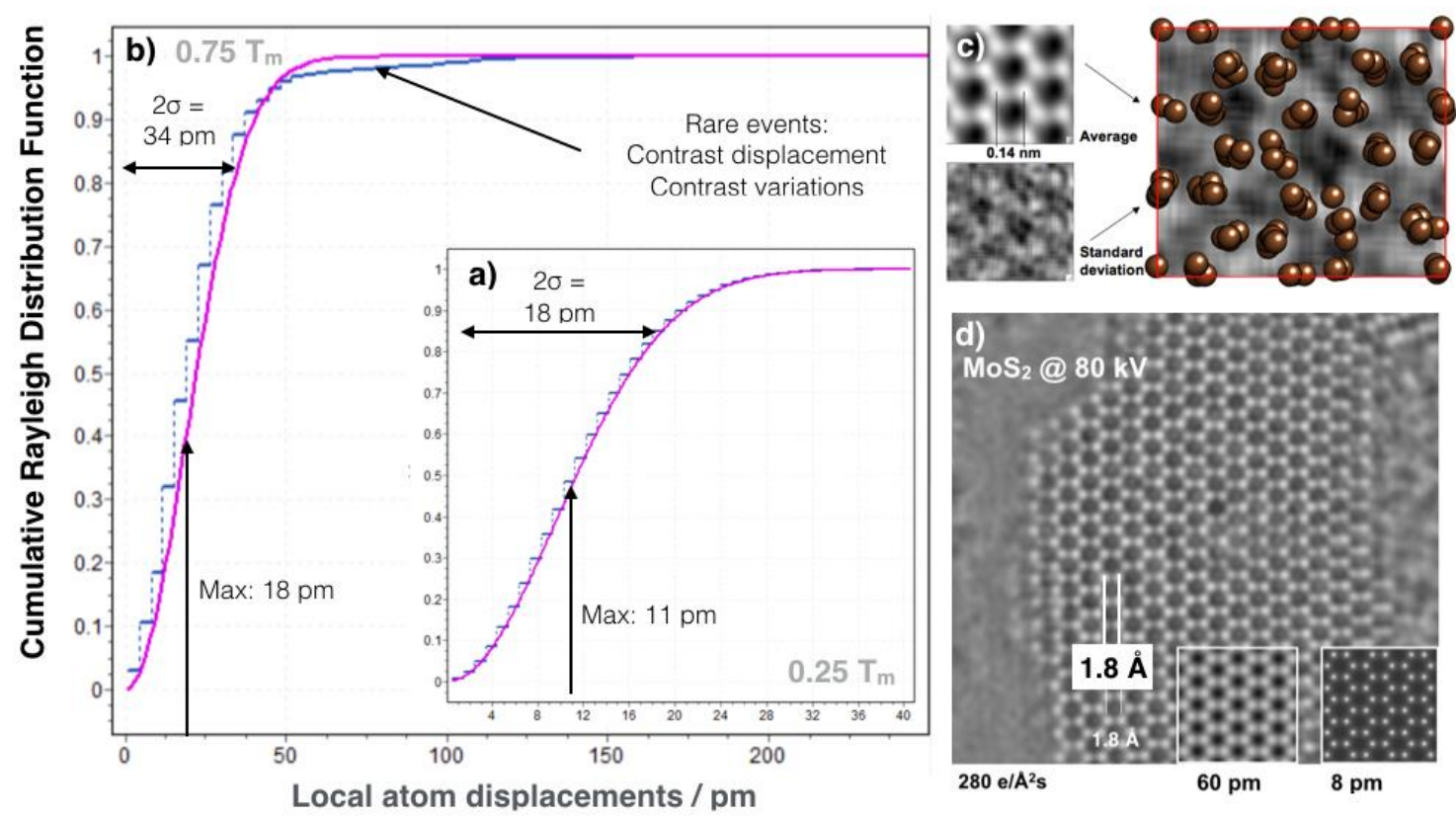

Figure 2. Studying beam induced atom dynamics: Large atom displacements in graphene $(a, b)$ that overwrite Debye-Waller factors [3] can be stimulated by the e-beam and are observed experimentally in $2 \mathrm{D}$ materials as contrast broadening, c) graphene, d) $\mathrm{MoS}_{2}$. 